\title{
Three dimensional electrode array for cell lysis via electroporation
}

\author{
Kuan-Ying Lu ${ }^{\text {a }}$, Andrew M. Wo ${ }^{\text {a, }}$, Ying-Jie Lo ${ }^{a}$, Ken-Chao Chen ${ }^{a}$, \\ Cheng-Ming Lin ${ }^{\mathrm{a}}$, Chii-Rong Yang ${ }^{\mathrm{b}}$ \\ a Institute of Applied Mechanics, National Taiwan University, Taipei 106, Taiwan \\ ${ }^{\mathrm{b}}$ Department of Mechatronic Technology, National Taiwan Normal University, Taipei 106, Taiwan
}

Received 7 March 2006; received in revised form 10 June 2006; accepted 9 August 2006

\begin{abstract}
Microfabricated devices for cell lysis have demonstrated many advantages over conventional approaches. Among various design of microdevices that employ electroporation for cytolysis, most utilize $\mathrm{Ag} / \mathrm{AgCl}$ wires or 2D planar electrodes. Although, simple in fabrication the electric field generated by $2 \mathrm{D}$ electrodes decays exponentially, resulting in rather non-uniform forcing on the cell membrane. This paper investigates the effect of electric field generated by 3D cylindrical electrodes to perform cell lysis via electroporation in a microfluidic platform, and compared with that by $2 \mathrm{D}$ design. Computational results of the electric field for both $2 \mathrm{D}$ and $3 \mathrm{D}$ electrode geometries showed that the 3D configuration demonstrated a significantly higher effective volume ratio-volume which electric field is sufficient for cell lysis to that of net throughflow volume. Hence, the efficacy of performing cell lysis is substantially greater for cells passing through 3D than 2D electrodes. Experimentally, simultaneous multi-pores were observed on leukocytes lysed with 3D electrodes, which is indicative of enhanced uniformity of the electric field generated by 3D design. Additionally, a single row of 3D electrode demonstrated a substantially higher lysing percentage (30\%) than that of 2D (8\%) under that same flow condition. This work should aid in the design of electrodes in performing cell lysis via electroporation.
\end{abstract}

(c) 2006 Elsevier B.V. All rights reserved.

Keywords: Cell lysis; Electrode design; Electroporation; Microfluidics

\section{Introduction}

Microfluidics has gained wide acceptance in bio/chemical studies in recent years (Bange et al., 2005; Pihl et al., 2005; Stone et al., 2004). As a result, the idea of packaging traditional laboratory instrumentation onto a chip - or lab-on-a-chip - is becoming a reality. Fluid transport and processing, sensing and/or actuation, and analysis elements in an integrated microfluidics platform are being accomplished with microfluidic components such as micropump, microvalve, micromixer, and microelectrodes (Isik et al., 2005) to perform specific tasks. The focus of this work is to utilize microelectrodes to perform cell lysis within a chip environment.

There are a number of approaches in performing lysis on the cell phospholipid membrane, with the predominant goal of extracting various elements - e.g. membrane and intracellular protein, RNA, and DNA - to study physiologic and pathologic

\footnotetext{
* Corresponding author. Tel.: +886 2 33665656; fax: +886 223639290 .

E-mail address: andrew @iam.ntu.edu.tw (A.M. Wo).
}

processes at the cellular level. These approaches can be classified into two groups: chemical and physical means (Graham and Rickwood, 1997). Chemical approaches mostly utilize lysis buffer with surfactant to solubilize the lipid membrane. However, the use of surfactant requires additional treatment to remove itself from the sample. Another main drawback is that although chemical methods are widely used for extraction of DNA, it is not ideal if the prime concern is integrity of protein, e.g. organelle (Lu et al., 2005). Moreover, in the limiting case of lysing a single cell picoliter volume of lysis solution in a microfluidic environment is needed (Irimia et al., 2004).

On the other hand, physical means of cell lysis employ a wide variety of approaches. Thermal method, in a reactor, is perhaps the most common (El-Ali et al., 2005; Lee et al., 2005), probably due to compatibility with PCR and DNA analyses. However, this may not be the method of choice for most protein analysis since protein denatures much easier than DNA in elevated temperature environment. Marentis et al. (2005) used energy from sound wave from a piezoelectric minisonicator to lyse leukemia HL-60 cells and Bacillus subtilis bacterial spores in microfluidic environment in the absence of chemical denaturants, enzymes or 
microparticles. Di Carlo et al. (2005) used poration of hydroxide ion through cell membrane for cell lysis. Marmottant and Hilgenfeldt (2003) demonstrated a novel approach in which controlled, oscillating microbubbles are sufficient to rupture lipid membranes.

Electroporation, another physical approach, is widely used to create transient pore through the membrane to deliver dyes, drugs, proteins, peptides, RNA, and DNA into cells (Neumann et al., 1989; Tsong, 1991). The technique applies an electric field which raises the transmembrane potential above a threshold (0.2-1.2 V) sufficient to disrupt the lipid by-layer and causes pores to form. In recent years, marriage of this technique with microfluidics to attain the same end has been widely accepted (Huang and Rubinsky, 2003; Lin and Huang, 2001; Lin et al., 2004; Shin et al., 2004), and was reviewed by Olofsson et al. (2003). Electroporation has been applied in microfluidic platform primarily to lyse the membrane permanently (Lee and Tai, 1999; Lu et al., 2005; Xia et al., 2005).

The design of electrodes is of crucial import in lysing cell, since the electrodes define the electric field. The majority of the effort thus far of utilizes simply with $\mathrm{Ag} / \mathrm{AgCl}$ wires (Gao et al., 2004; Khine et al., 2005; Lee and Tai, 1999; Xia et al., 2005) or microfabricated planar electrode design (Lee and Tai, 1999). The drawback for planar electrodes is the exponential decay of the electric field away from the plane of the electrode. With cell dimension typically not much greater than the channel height, a cell would experience large non-uniformity in the electric field during electroporation. Very limited studies utilize 3D electrodes for cytolysis. Lu et al. (2005), for example, successful employed pointed, 3D electrodes with the primary intent of preserving organelle integrity. However, the efficacy of electrode design in lysing cells - not being the focus of $\mathrm{Lu}$ et al. (2005) - was not investigated.

This paper aims to quantify the effect of utilizing 3D electrodes (Fig. 1a) with the aim of enhancing cell lysis via electroporation in a microfluidic platform. Both computation and experimental efforts using leukocytes were undertaken, with comparison made to planar electrode design. Results from this work should enhance design of electrodes to perform cell lysis using electroporation.

\section{Electroporation}

The phenomenon utilizes electric field to create transient or permanent pore (s) on the cell membrane. The phenomenon occurs when sufficient voltage required for dielectric breakdown of the membrane, about $0.2-1.5 \mathrm{~V}$, is imposed by an external electric field (Khine et al., 2005). This transmembrane potential $\Delta \varphi$ for a spherical cell can be expressed as (Neumann et al., 1989),

$\Delta \varphi(t)=1.5 a E \cos \theta\left(1-\exp \left(\frac{-t}{\tau_{\mathrm{m}}}\right)\right)$,

where $a$ is the radius of cell, $E$ the external electric field, $\theta$ the polar angle measured from the center of the cell with respect to the direction of electric field, and $\tau_{\mathrm{m}}$ is the charging time of the cell membrane. When the transmembrane potential is
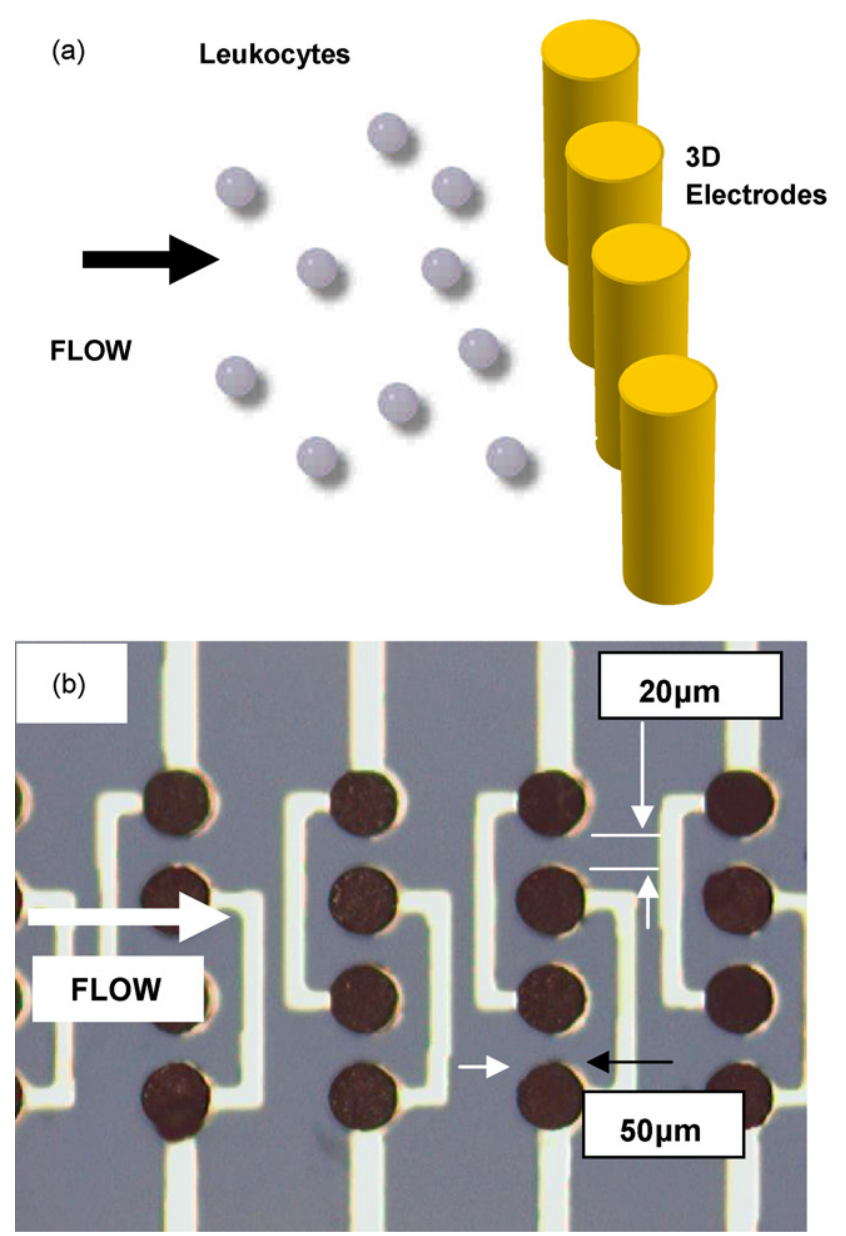

Fig. 1. Three dimensional cylindrical electrodes. (a) Sketch of leukocytes approaching a singe row of electrode. (b) Micrograph of fabricated device. The diameter of each electrode is $50 \mu \mathrm{m}$ and spacing between electrodes is $20 \mu \mathrm{m}$. The height of each electrode is $50 \mu \mathrm{m}$.

strong enough, pores will be formed. In general, pores can be of two states: reversible electrical breakdown, with the pores reseal themselves when the external electric field is removed, and irreversible breakdown, where the damage is permanent. The signal of electroporation is usually in the form of pulses in order to avoid damaging DNA or organelles, with the pulse duration between 10 and $1000 \mu \mathrm{s}$. The charging time of cell membrane could be shown as (Muller et al., 2001):

$\tau_{\mathrm{m}}=a C_{\mathrm{m}}\left(\frac{1}{\sigma_{\mathrm{i}}}+\frac{1}{2 \sigma_{\mathrm{e}}}\right)$,

where $C_{\mathrm{m}}$ is the membrane capacitance, $\sigma_{\mathrm{i}}$ intracellular conductivity, and $\sigma_{\mathrm{e}}$ is conductivity of the external medium. If cells are embedded in high conductivity medium, the charging time will be shorter. For most mammalian cells, the charging time is quite short-on the order of several hundreds nano seconds (Muller et al., 2001). Therefore, pulses of sufficiently long duration (100 $\mu$ s used here), compared to the membrane charging time, behaves as if the pulse excitation is DC. Hence, the reduced form of Eq. (1) can be used instead, namely,

$\Delta \varphi \approx 1.5 a E \cos \theta$

can be used to characterize the transmembrane potential. 


\section{Computation and experimental aspects}

\subsection{Computation}

Computational effort was undertaken to aid the design of the microdevice in characterizing the electric field prior to fabrication. The commercial code Comsol Multiphysics (Comsol Group) was used to compute the electric field of planar (pointed and circular) and 3D electrodes (cylinders). The computational domain of the 3D configuration covered a region of $290 \mu \mathrm{m}$ width (cross stream dimension), which contained the extent of four cylinders. The streamwise extent was $250 \mu \mathrm{m}$, which included only one row of electrodes. The computation was conducted to a height of $170 \mu \mathrm{m}$ (actual device channel height is $50 \mu \mathrm{m}$ ) to ensure that sufficient decay in the electric field had indeed taken place. Boundary conditions were: vanishing gradient of the electric potential normal to the surface at all locations except at the electrode surface. On the electrodes, the voltage prescribed was a constant $10 \mathrm{~V}$. The spatial increment of the computational grid was $1 \mu \mathrm{m}$ uniformly.

We also calculated the effective volume ratio of the three electrode geometries under different applied voltages and channel heights. This ratio is defined as the volume of throughflow with transmembrane potential sufficient for cytolysis to the total volume that the fluid occupied. For the 3D electrode configuration the total volume does not include the volume of the cylinders. In other words, this ratio provides an idea of the efficacy of cell lysis as cells flow through the region with electrodes within the microchannel. Using a typical transmembrane potential of $1 \mathrm{~V}$, the threshold electric field strength for cell lysis was estimated using Eq. (3) for membrane thickness of approximately $5 \mathrm{~nm}$ and resulted at $2000 \mathrm{kV} / \mathrm{cm}$ (Tsong, 1991). Using this value and results from computation of electric field for various electrode geometries, calculations for the effective volume ratio were made for channel height of 50 and $100 \mu \mathrm{m}$.

\subsection{Fabrication}

Fabrication process of the device consists of fabrication of the planar electrodes, 3D electrodes, and the microchannel. Each component is described in some detail below.

The planar electrodes was made via conventional MEMS approach as follows. First, $\mathrm{Au} / \mathrm{Cr}(200 / 30 \mathrm{~nm})$ was deposited on glass substrate by electron beam evaporation. Positive photoresist (PR) (model S1813, Shipley) were spincoated on the metal layer, then exposed to UV light through a patterned transparency mask, followed by development of the PR. Then, the PR was stripped leaving the patterned electrodes. Teflon was applied, as protection for electrodes from corrosion due to applied pulses, using the following three-step procedure. First, a layer of PR was spincoated and, after exposure to UV light through a masked pattern and subsequent development, only the portion above the metal layer was removed. Second, Teflon was spincoated onto the bare metal and the remaining PR (between electrodes). Finally, lift-off process was performed on the PR, leaving the Teflon to protect the metal layer only.
To fabricate the 3D electrodes, first, a thin metal layer with the same area as the base area of the 3D cylindrical electrodes was formed for subsequent electroforming of the $3 \mathrm{D}$ electrode structure. This metal "seed layer" had identical fabrication steps as that of depositing bare metal layer on the substrate of the planar electrodes described earlier (via electron beam evaporation). The fourth step fabricated the SU-8 mold in which the 3D electrodes would electroform inside. The recipe for SU-8 structure was as follow. It was spun at $4000 \mathrm{rpm}$ for $30 \mathrm{~s}$, which corresponded to a height of $70 \mu \mathrm{m}$ even though the height of $3 \mathrm{D}$ electrodes was $50 \mu \mathrm{m}$. The reason for the over-height of the mold was to avoid over-grown of copper during the electroforming process, which would also cause a detrimental short circuit. After spinning, the SU8 was soft baked at $65^{\circ} \mathrm{C}$ for $8 \mathrm{~min}$ and $95^{\circ} \mathrm{C}$ for $25 \mathrm{~min}$. After exposure for $33 \mathrm{~s}$ in $14 \mathrm{~mJ} / \mathrm{cm}^{2}$ power of UV light, post-exposure bake was made at $65^{\circ} \mathrm{C}$ for $2 \mathrm{~min}$ and $95^{\circ} \mathrm{C}$ for $7 \mathrm{~min}$, followed by development with agitation aided by ultrasound.

Then, electroforming was employed to fabricate the 3D copper electrodes. The process is very similar to electroplating with two differences. First, electroforming is normally used in thick deposition (tens of micron) and, second, the deposited structure can be made to conform to a mold. Hence, the topological accuracy of the 3D electrodes by electroforming is essentially determined by the quality of the SU-8 mold. If a crack underneath the SU-8 structure exists the electroforming solution will permeate into the crack and metal will be deposited, which might cause a detrimental short circuit between adjacent electrodes. The copper electrodes were fabricated using a sulfate-based copper electrolytic bath. The plating bath contained $200 \mathrm{~g} / \mathrm{l}$ copper sulfate $\left(\mathrm{CuSO}_{4} \cdot 5 \mathrm{H}_{2} \mathrm{O}\right), 85 \mathrm{~g} / \mathrm{l}$ sulfuric acid $\left(\mathrm{H}_{2} \mathrm{SO}_{4}\right)$, and $50 \mathrm{mg} / \mathrm{l}$ chloride ion $\left(\mathrm{Cl}^{-}\right)$operating at room temperature and $\mathrm{pH}$ below 1 . The content of the bath was kept clean by circulation and agitation by pumping through a filter with $0.1 \mathrm{~mm}$ pores. Small quantity of surfactant was added to make the electrolyte more hydrophilic and the electroformed copper brighter. The pre-treatment of the photoresist mold and the process control of electroplating deserved attention to ensure that metallic microstructures of high quality can be obtained. Metal was deposited at a current density of $1 \mathrm{~A} / \mathrm{dm}^{2}$ for about $4 \mathrm{~h}$. Next, the SU-8 mold was removed with Remover PG (Micro Chem Corp.) at $80^{\circ} \mathrm{C}$ under ultrasonic agitation. Teflon was also used to protect the electrodes with the same procedure as that of the planar electrodes. Fig. 1b shows the fabricated 3D electrodes.

The microchannel was fabricated with (poly)dimethylsiloxane (PDMS) using conventional soft lithographic process (Xia and Whitesides, 1998). SU-8 was used in forming the master mold for the microchannel with $50 \mu \mathrm{m}$ height, $290 \mu \mathrm{m}$ width, and about $1 \mathrm{~cm}$ length. Replica molding technique for the PDMS elastomer allowed the microchannel geometry to take shape. Oxygen plasma treatment was applied on both the molded PDMS microchannel and the substrate with electrodes to allow irreversible bonding of the two parts to form the encapsulated device. Finally, inlet and outlet ports were inserted for pumping with syringe. 


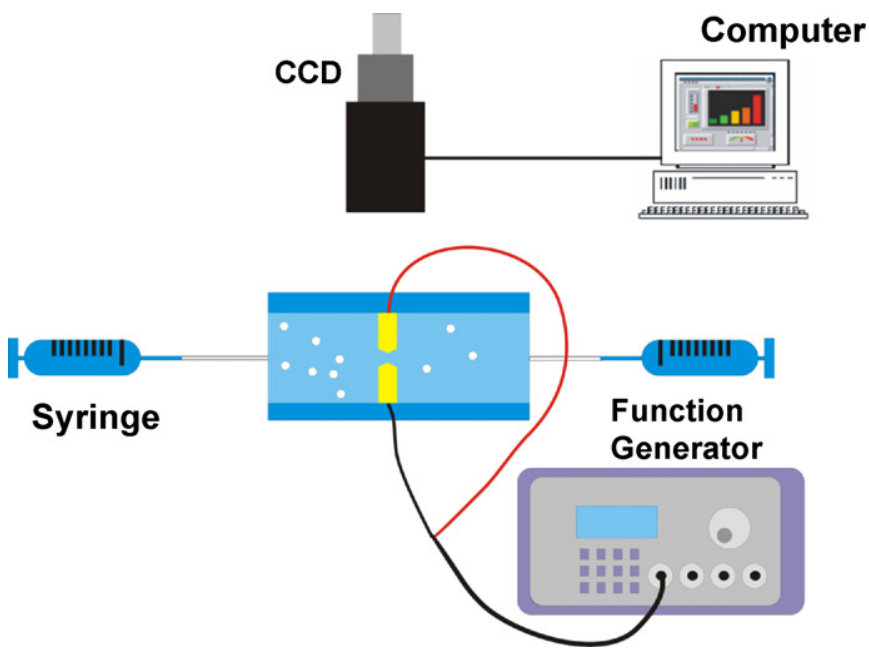

Fig. 2. Sketch of the entire experimental setup with the cell lysis microdevice. Syringe was used to pump the leukocytes through the device with $10 \mathrm{~V}, 100 \mu \mathrm{s}$ pulse supplied from a function generator. Images were captured by CCD for post-processing.

\subsection{Experimental setup}

Leukocytes (Chinese Blood Donation Association) were diluted with physiological saline solution in ratio of 1:100 by volume. Then, the cells were injected into the straight microchannel by a syringe pump at the inlet and collected at the outlet. Excitation to the electrodes was applied with $10 \mathrm{~V}$, $100 \mu$ s pulse at $4 \mathrm{~Hz}$ using a function generator (model 195; Wavetek Universal). Micrograph images were recorded by CCD camera, and stored for data interrogation. Fig. 2 shows the experimental setup.

Trypan blue was used to verify successful cytolysis. Khine et al. (2005) also used Trypan blue dye, and independently with
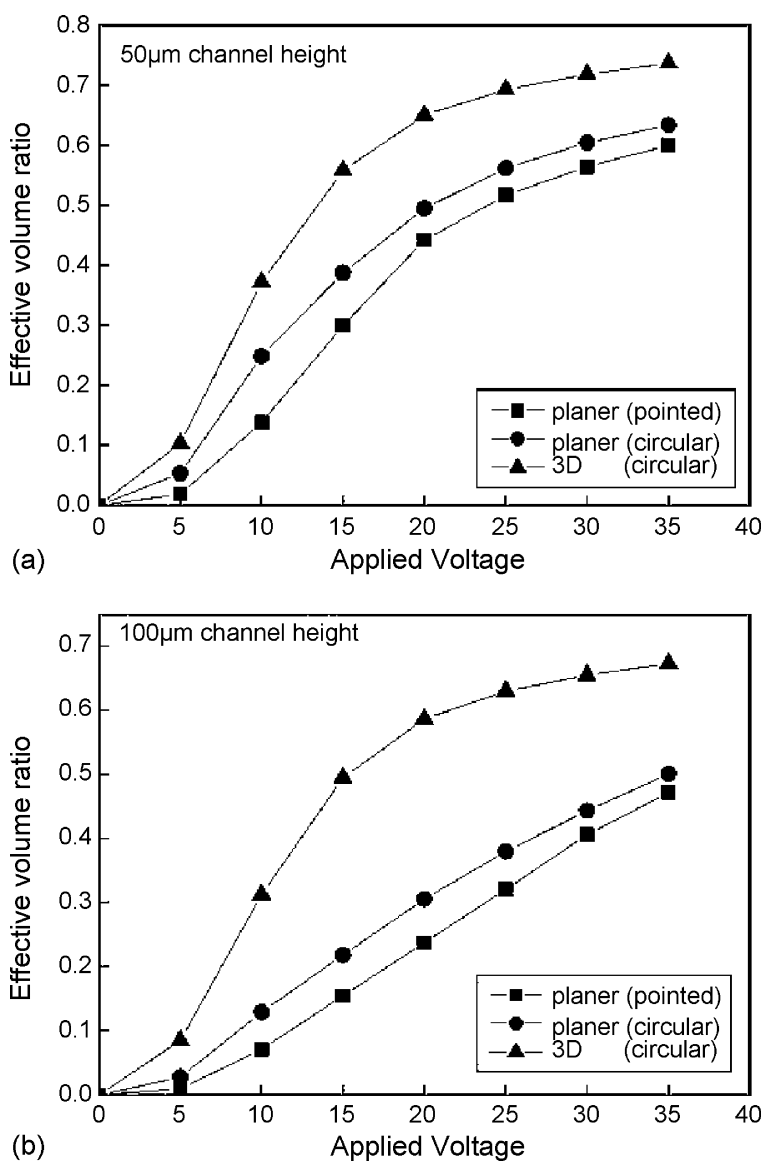

Fig. 4. Computational results of the effective volume ratio defined as volume of throughflow with transmembrane potential sufficient for cytolysis to the total throughflow volume. Applied voltage of $0-35 \mathrm{~V}$ and channel heights of: (a) $50 \mu \mathrm{m}$ and (b) $100 \mu \mathrm{m}$ are computed. Results show the effective volume ratio for $3 \mathrm{D}$ electrodes is larger than that of either pointed or circular planar electrodes considered.
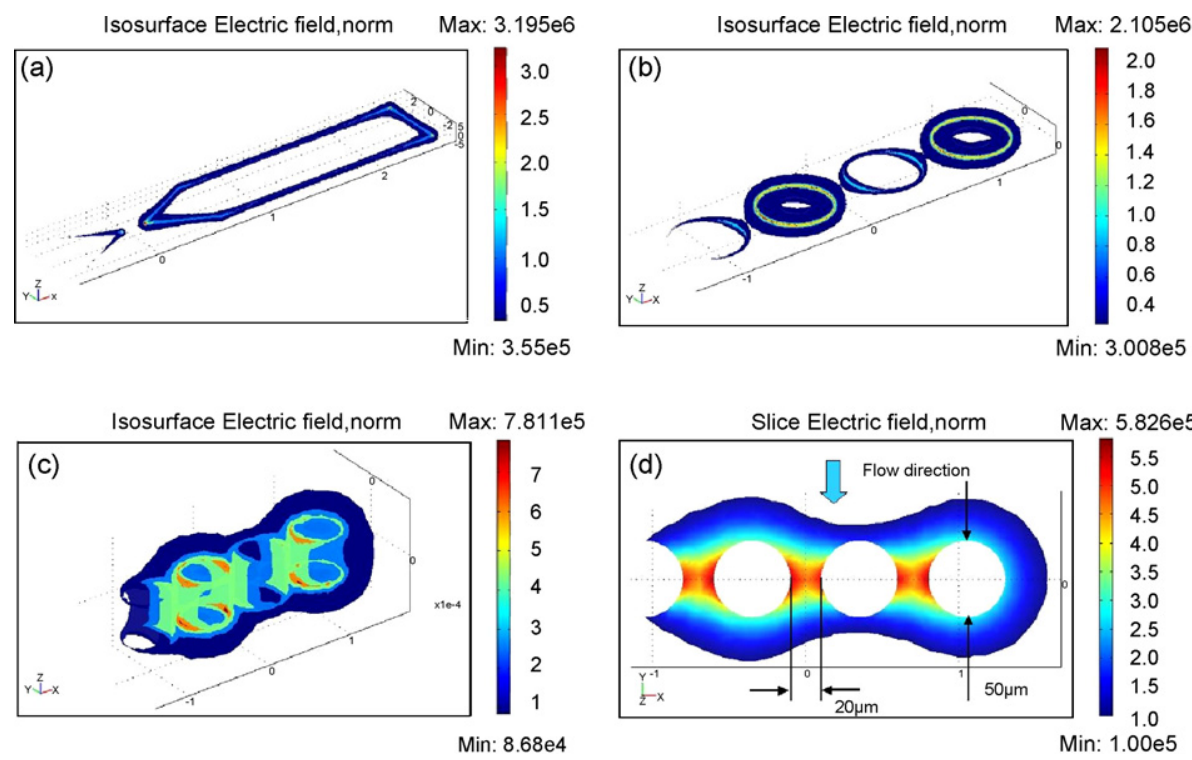

Fig. 3. Computed iso-electric field contour of three electrode configurations: (a) 2D pointed planar, (b) 2D circular planar, and (c) 3D cylindrical electrodes, with mid-height plane $(z=25 \mu \mathrm{m})$ contour shown in (d). The applied voltage was $0 \mathrm{~V}$ and, at the adjacent electrode, $10 \mathrm{~V}$ for all cases. Results show that the electric field decays rapid away form the electrodes for the planar cases, ( $a$ and $b$ ), but does not decay much with height for the 3D electrode. Results at mid-height of 3D electrode, (d), is representative of that at other heights since (c) confirms that the electric field is almost invariant along the cylindrical axis. 
cytoplasmic dye Calcein AM, to definitively identify the lysed cells. Although there are natural pores on cell membrane, exclusion measurement of Trypan blue is routinely used as a test of cell viability since healthy membrane would prevent seepage of the toxin. Once the membrane is made permeable (via electroporation in this work) the dye could accumulate within the cell staining the intracellular elements a distinct bluish color. In the event that the membrane disintegrated, location of the color change (turned bluish) would be indicative of that of the intracellular elements.

\section{Results and discussion}

\subsection{Computation of the electric field}

Fig. 3 shows results of the computed iso-electric field surfaces for the two planar electrodes - pointed (Fig. 3a) and circular (Fig. 3b) - and the 3D electrodes (Fig. 3c and d). Both Fig. 3a and b suggest that large spatial gradient exists near the $2 \mathrm{D}$ electrodes as indicated by large values (non deep blue colored area) near the edge of the electrodes and decay rapidly away. For the 3D cylindrical electrodes (Fig. 3c), even though there exists some variation near the top and bottom of the electrodes - as can be expected due to edge effect - the electric field predominantly does not decay much over the entire height. Fig. 3d plots the electric field distribution at the mid-height $(25 \mu \mathrm{m})$ plane of the cylinder (or mid-height plane of Fig. 3c), which is representative of the electric field at other heights except very close to the end regions.
As a cell passes through the area between the cylindrical electrodes it enters into a region of approximately $20 \mu \mathrm{m}$ width of high field strength area, as indicated in Fig. 3d. The extend of this region is large enough such that it can easily encompasses the entire leukocyte ( $10 \mu \mathrm{m}$ in diameter). With sufficient electric field strength, gradient of transmembrane potential is setup to accomplish cytolysis in a fashion where the cell would experience much more uniform distribution of field strength than that under planar electrodes.

Fig. 4 presents the results of effective volume ratio, as defined previously. Results show this ratio for the $3 \mathrm{D}$ electrode is larger than planar electrodes in all cases. Moreover, at a particular applied voltage, lysing effectiveness decreases as the channel height increases for the planar electrodes. This overall trend is indicative of the rapid decay nature of planar design, where the electric field of the 3D design essentially remains constant over the entire height. Although the planar electrodes are not much worse off than the 3D design at $50 \mu \mathrm{m}$ channel height, the difference in lysing effectiveness between the two designs increases as the channel height increases.

Lysing events in the microdevice are presented in Fig. 5 for the pointed, planar electrodes and Fig. 6 for 3D electrodes. We adopted two independent approaches in verifying that the membrane was indeed lysed. First, when the optical appearance of a cell turns from a normal cell with intact membrane to that of a "ghost cell" this would be indicative that its membrane is no longer acting as a boundary of intracellular elements and thus begins to disintegrate. Lu et al. (2005) also employed this approach, which proved to be effective in deciphering lysed
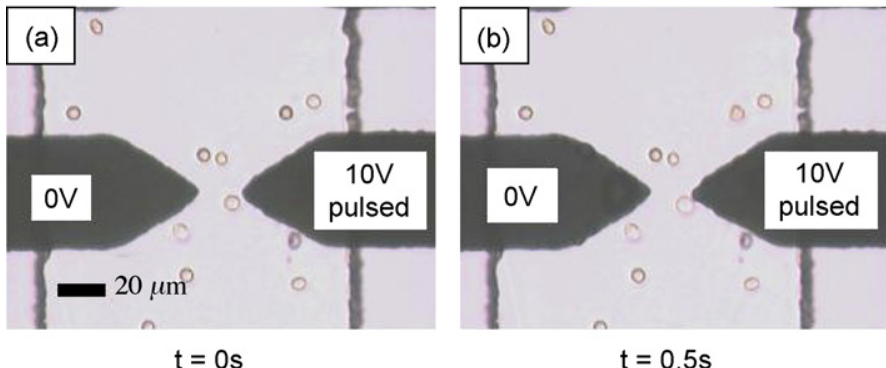

$t=0.5 s$

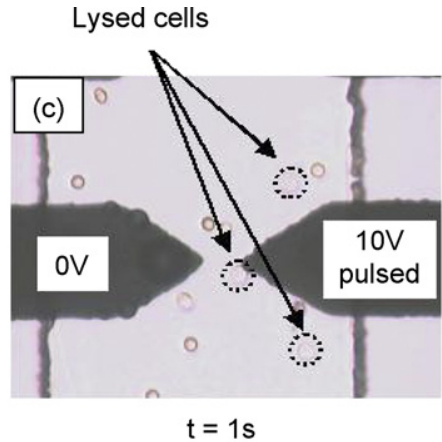

Lysed cells

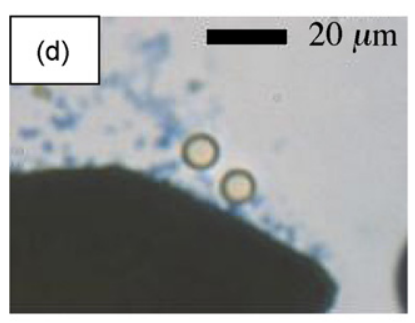

$\mathrm{t}=0 \mathrm{~s}$

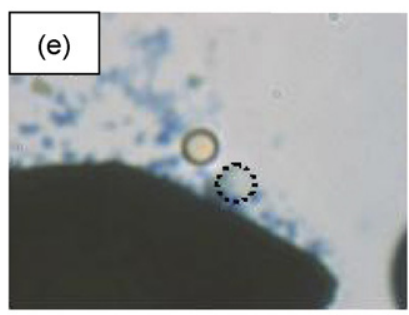

$t=2 s$

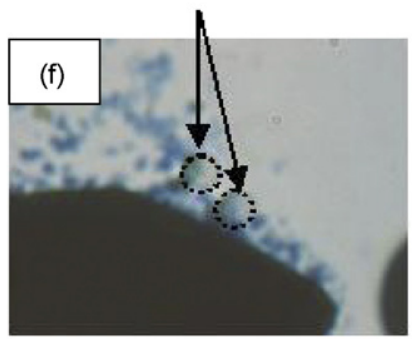

$t=16 s$

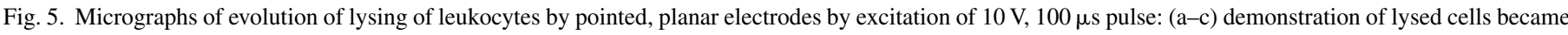

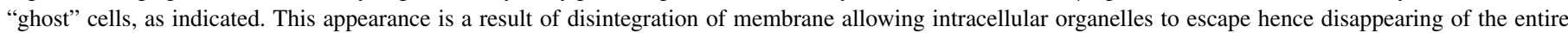

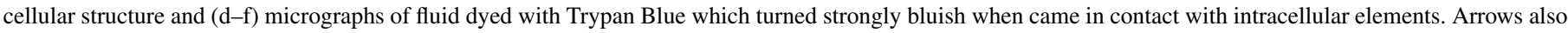
confirmed disappearing of the cellular structure. 


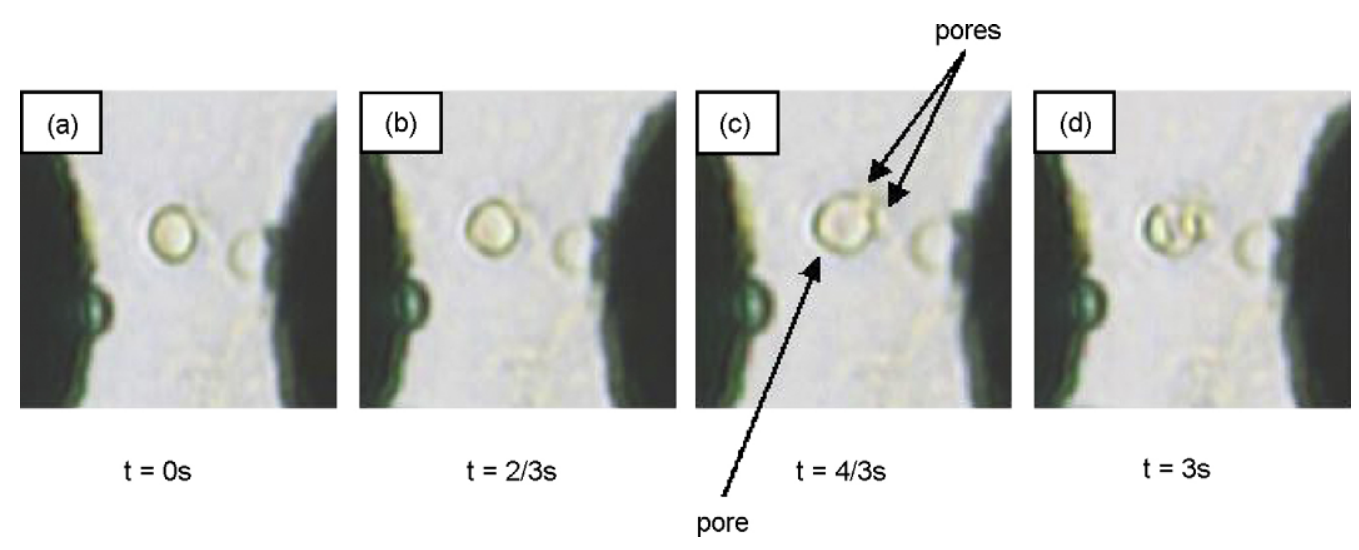

Fig. 6. Micrographs of time evolution of leukocytes being lysed by 3-D electrodes under excitation of $10 \mathrm{~V}, 100 \mu$ s pulse (same as that in Fig. 5). Graduate disintegration of the cellular structure (becoming "ghost" cells) is indicative of successful cytolysis. Moreover, simultaneous multiple pores on the cell membranes were observed as shown by arrows.

cells. Second, Trypan blue was used, with the aforementioned function (see Section 3.3). Fig. 5 presents a series of events of leukocytes closed to the planar electrodes being lysed. The events in Fig. 5a-c showed some cells were being turned into ghost cells, with their membrane gradually opening and fainting within a few seconds. In the events here, the entire duration for lysis was about $1 \mathrm{~s}$. Long time observation of these cells confirmed that they eventually disappeared. Fig. 5d-f used Trypan blue to locate the intracellular elements. Results suggested the cells closed to the electrode surface, as observed, were being definitively lysed. Staining here was indicative that portions of the intracellular element were located outside the disintegrated membrane. In this case, the entire lysis process occurred in about $16 \mathrm{~s}$. The difference in the duration of lysis between that from Fig. 5a-f was likely due to the difference in height from the electrode surface for the two cases, indicative that the exponential decay nature of the electric field causing substantial difference in lysing results.

Lysis of leukocytes with the 3D electrodes demonstrated marked difference from that of the planar geometry. As cells flow past the electrodes, Fig. 6 shows simultaneous multiple pores were observed during the lysis process. This is likely due to that the cell was exposed to a more uniform electric field, as suggested by results of Fig. $3 \mathrm{~d}$ and discussed earlier. The enhanced uniformity in electric field, as compared to that of produced by planar electrodes, apparently caused multiple locations of membrane to exceed that of the transmembrane potential simultaneously and resulted in that of Fig. 6 . The lysis process required about three seconds.

The efficacy of cytolysis was further quantified by comparing the lysis percentage of both 2D (pointed) and 3D electrode design. This percentage is defined as the amount of lysed cells per total number of cells passing through a single row of electrode. This was done by image post-processing in counting the number of lysed cells for both devices under approximately the same background flow rate. Results show that for 2D electrodes, the lysing percentage was $8.0 \pm 6 \%$ for flow rate of $57 \mu \mathrm{m} / \mathrm{s}$. For 3 D electrodes, the lysing percentage was $30.5 \pm 5 \%$ for flow rate of $50 \mu \mathrm{m} / \mathrm{s}$. This result suggests that lysing effect of 3D elec- trodes indeed enable cell lysis much more pronouncedly than that of planar electrodes.

\section{Conclusions}

Electroporation - a dominant method to transfer DNA and other genetic substance into the cell - has been utilized in this work to investigate the efficacy of cell lysis. The focus is the characterization of electric field generated by electrodes with planar and 3D cylindrical geometries in a microfluidic device. Computational results show that the effective lysis volume ratio of the 3D electrode is higher than that of the planar design. The primary reason being, unlike the planar electrodes, the electric field of the 3D electrodes does not decay rapidly away from the substrate, resulting in much greater effective volume. Experimentally, leukocytes were lysed successfully in seconds by both types of electrode designs, and intracellular elements were extracted through cell membrane as observed by increased intensity of Trypan blue. Simultaneous multiple pores were observed on leukocyte lysed with 3D electrodes and just single pore with planar electrodes. This is believed to be due to more uniform electric field of 3D geometry than that of planar geometry.

Much improved lysing percentage for 3D electrodes (30\%) compared to planar design (8\%) carries great design implication. If one desires to increase lysing percentage for 3D design to, say, $90 \%$, one can simply increase the number of rows of electrode that the cells would pass through to three or four. In contrast, many more pairs of planar electrodes are needed to achieve the near-complete lysing process. Lu et al. (2005) used an estimated 30 sets of pointed 3D electrodes to perform cell lysis as a cell progressed downstream-although the actual number of pairs of electrodes to complete lysis was not evident. Over-lysing a cell would conceivably damage the intracellular elements. Since cell lysis is a process with its end goal being extraction of these elements, gene transfection, or the like, the process should be performed to a degree just suffice for the specific application. Hence, this work should aid in the design of electrodes in performing cell lysis via electroporation in a particular application. 


\section{Acknowledgements}

The authors are grateful for partial financial support from the National Science Council (Grant NSC 94-2212-E-002-054) and the Ministry of Economic Affairs.

\section{References}

Bange, A., Halsall, H.B., Heineman, W.R., 2005. Biosens. Bioelectron. 20 (12), 2488-2503.

Di Carlo, D., Ionescu-Zanetti, C., Zhang, Y., Hung, P., Lee, L.P., 2005. Lab Chip 5 (2), 171-178.

El-Ali, J., Gaudet, S., Gunther, A., Sorger, P.K., Jensen, K.F., 2005. Anal. Chem. 77 (11), 3629-3636.

Gao, J., Yin, X.F., Fang, Z.L., 2004. Lab Chip 4 (1), 47-52.

Graham, J.M., Rickwood, D., 1997. Subcellular Fractionation: A Practical Approach. Oxford University Press, New York.

Huang, Y., Rubinsky, B., 2003. Sens. Actuators A Phys. 104 (3), 205-212.

Irimia, D., Tompkins, R.G., Toner, M., 2004. Anal. Chem. 76 (20), 6137-6143.

Isik, S., Berdondini, L., Oni, J., Blochl, A., Koudelka-Hep, M., Schuhmann, W., 2005. Biosens. Bioelectron. 20 (8), 1566-1572.

Khine, M., Lau, A., Ionescu-Zanetti, C., Seo, J., Lee, L.P., 2005. Lab Chip 5 (1), $38-43$.
Lee, C.Y., Lee, G.B., Lin, J.L., Huang, F.C., Liao, C.S., 2005. J. Micromech. Microeng. 15 (6), 1215-1223.

Lee, S.W., Tai, Y.C., 1999. Sens. Actuators A Phys. 73 (1-2), 74-79.

Lin, Y.C., Huang, M.Y., 2001. J. Micromech. Microeng. 11 (5), 542-547.

Lin, Y.C., Li, M., Wu, C.C., 2004. Lab Chip 4 (2), 104-108.

Lu, H., Schmidt, M.A., Jensen, K.F., 2005. Lab Chip 5 (1), 23-29.

Marentis, T.C., Kusler, B., Yaralioglu, G.G., Liu, S., Haeggstrom, E.O., KhuriYakub, B.T., 2005. Ultrasound Med. Biol. 31 (9), 1265-1277.

Marmottant, P., Hilgenfeldt, S., 2003. Nature 423 (6936), 153-156.

Muller, K.J., Sukhorukov, V.L., Zimmermann, U., 2001. J. Membr. Biol. 184 (2), 161-170.

Neumann, E., Sowers, A.E., Jordan, C.A.E., 1989. Electroporation and Electrofusion in Cell Biology. Plenum Press, New York.

Olofsson, J., Nolkrantz, K., Ryttsen, F., Lambie, B.A., Weber, S.G., Orwar, O., 2003. Curr. Opin. Biotechnol. 14 (1), 29-34.

Pihl, J., Karlsson, M., Chiu, D.T., 2005. Drug Discov. Today. 10 (20), 1377-1383.

Shin, Y.S., Cho, K., Kim, J.K., Lim, S.H., Park, C.H., Lee, K.B., Park, Y., Chung, C., Han, D.C., Chang, J.K., 2004. Anal. Chem. 76 (23), 7045-7052.

Stone, H.A., Stroock, A.D., Ajdari, A., 2004. Ann. Rev. Fluid Mech. 36 381-411.

Tsong, T.Y., 1991. Biophys. J. 60 (2), 297-306.

Xia, F.Q., Jin, W.R., Yin, X.F., Fang, Z.L., 2005. J. Chromatogr. A 1063 (1-2), 227-233.

Xia, Y.N., Whitesides, G.M., 1998. Ann. Rev. Mater. Sci. 28, 153-184. 